\title{
1 Evolutionary rate shifts suggest species-specific adaptation events in HIV-1 and SIV
}

$4 \quad$ Maoz Gelbart and Adi Stern

5 School of Molecular Cell Biology and Biotechnology, George S. Wise faculty of Life Sciences, Tel Aviv

6 University, Israel

$7 \quad *$ Correspondence to AS: sternadi@post.tau.ac.il

8 Abstract

9 The process of molecular adaptation following a cross-species virus transmission event is

10 currently poorly understood. Here, we identified 137 protein sites that experienced deceleration

11 in their rate of evolution along the HIV-1/SIV phylogeny, likely indicating gain-of-function and

12 consequent adaptation. The majority of such events occurred in parallel to cross-species

13 transmission events and varied between HIV-1 groups, indicating independent adaptation

14 strategies. The evolutionary rate decelerations we found were particularly prominent in

15 accessory proteins that counteract host antiviral restriction factors, suggesting that these factors

16 are a major barrier to viral adaptation to a new host. Surprisingly, we observed that the non-

17 pandemic HIV-1 group 0 , derived from gorillas, exhibited more rate deceleration events than

18 the pandemic group $\mathrm{M}$, derived from chimpanzees. We suggest that the species barrier is higher

19 when the genetic distance of the hosts increases. Our approach paves the way for subsequent

20 studies on cross-species transfers in other major pathogens. 


\section{Introduction}

22 The Human Immunodeficiency Viruses HIV-1 and HIV-2 are the causative agents of AIDS in

23 humans, infecting millions of people worldwide. Both viruses emerged from a clade of

24 lentiviruses known as the Simian Immunodeficiency Virus (SIV), which naturally infect a variety

25 of non-human primate species. HIV in humans arose from several independent transmission

26 events of primate SIVs that resulted in HIV-1 groups M and N (from SIV infecting chimpanzees,

27 SIVcpz), HIV-1 groups O and P (from SIV infecting gorillas, SIVgor), and HIV-2 groups A through $\mathrm{H}$

28 (from SIV naturally infecting sooty mangabeys, SIVsmm) [1-3]. The gorilla infecting lentivirus,

29 SIVgor, is itself a result of a transmission of SIVcpz to gorillas [4, 5]. Phylogenetic analyses date

30 the most common recent ancestors of HIV groups $\mathrm{M}$ and $\mathrm{O}$ to the beginning of the $20^{\text {th }}$ century,

31 making it a relatively new human pathogen $[6,7]$. Similar analyses of SIVgor date the inception

32 of this virus in the western lowland gorilla population somewhere in the $19^{\text {th }}$ century [4]. SIVcpz

33 itself was found to be a transmission from other primates, dated at roughly 1500 and leading to

34 the two lineages of SIVcpz: SIVcpzptt infecting the chimpanzee subspecies Pan troglodytes

35 troglodytes of central Africa, and SIVcpzpts infecting the Pan troglodytes schweinfurthii

36 chimpanzee subspecies of eastern Africa [5, 8-12].

37 How viruses are able to cross species barrier is a subject of much interest, since many pandemic

38 human viruses arose from zoonosis events such as the influenza strain of "Spanish flu" H1N1,

39 measles virus (MeV), and SARS coronavirus [13]. Due to genetic differences between the hosts,

40 virus adaptation occurs at multiple levels: at the level of entry to target cells; interaction with

41 the host adaptive immune system; interaction with host antiviral restriction factors; and

42 recruitment of host cellular machinery by the virus [14-16]. Protein adaptations are reflected in

43 the history of genomes and may be manifested in changes in the amino acid substitution rates

44 of the adaptive sites, which are expected to be more conserved in the clade where adaptation 
45 happened [17]. This is due to new roles gained by these protein sites that constrain their

46 evolution. A reciprocal phenomenon where amino acids are less conserved in one clade than in

47 other clades is also possible, and may either reflect a loss of function or a gain of function

48 manifested as positive diversifying selection. Identification of such "rate shifting sites" can thus

49 reveal virus adaptation events, and is expected to promote a better understanding of cross-

50 species transmissions in general and in the HIV pandemic in particular in this study.

51 A method for identification of evolutionary rate changes has been previously established and

52 was used to study intra-subtype HIV adaptation events [18]. To date, the lack of SIVcpz and

53 group O full genomic sequences limited the ability to study adaptation across cross-species

54 transmission events. Here, we utilized the growing availability of diverse and full HIV-1 and SIV

55 genomes to identify many sites in various SIVcpz/SIVgor/HIV-1 clades whose evolutionary rate

56 changed across clades [19]. We demonstrate how cross-species transmissions are correlated

57 with abundant evolutionary rate shifts and how known adaptation events are manifested in

58 different amino acid substitution rates between lineages. Based on the rate shift patterns, we

59 suggest previously unknown adaptation events, and highlight the exceptional amount of

60 evolutionary rate shifts observed in HIV-1 group O, possibly due to a more extreme host species

61 barrier.

62 Results

63 We have previously developed the RASER tool to identify sites that display change in the rate of

64 evolution along a given branch of a phylogenetic tree (see example in Figure 1) [18]. In essence,

65 this tool takes a phylogeny and a multiple sequence alignment and contrasts the rates of

66 evolution of amino acids along all branches in the phylogeny, to determine if the evolutionary

67 rates of some amino acids in some branches are better explained by a model that allows for 
68 evolutionary rate changes. We queried the Los Alamos HIV database [19] for all available HIV-1,

69 SIVcpz and SIVgor full sequences, from which we took the sequences of all nine HIV-1 proteins.

70 Due to high representation of group $M$ sequences, we downsampled this group to the size of

71 available group $\mathrm{O}$ sequences, retaining the strains with the most internal variation (total number

72 of sequences $\mathrm{N}=126$, see Methods). A different downsample of group $\mathrm{M}$ sequences to a bigger

73 size was also conducted, to validate results robustness to cohort size variation ( $N=223$, see

74 Methods). All site coordinates are reported based on the HIV-1 subtype B reference strain HXB2

75 (Methods).

76 Most rate shift events were identified in speciation branches. We first sought to characterize

77 the branches where rate shift events were suggested. A total of 271 rate shifting positions were

78 identified along the phylogeny. 137 of them were identified as rate-deceleration events and an

79 additional 134 were identified as rate-accelerations. As listed in Table 1, 230 out of the 271 rate

80 shifting positions were attributed to four branches: the branch separating group M from SIVcpz,

81 the branch separating group O from SIVgor, the branch separating SIVgor and group O from

82 SIVcpz and the branch separating SIVcpzpts from other viruses. Likelihood ratio tests were found

83 to be highly significant in favor of a rate shift model across all HIV proteins (Supplementary

84 Table 2). 


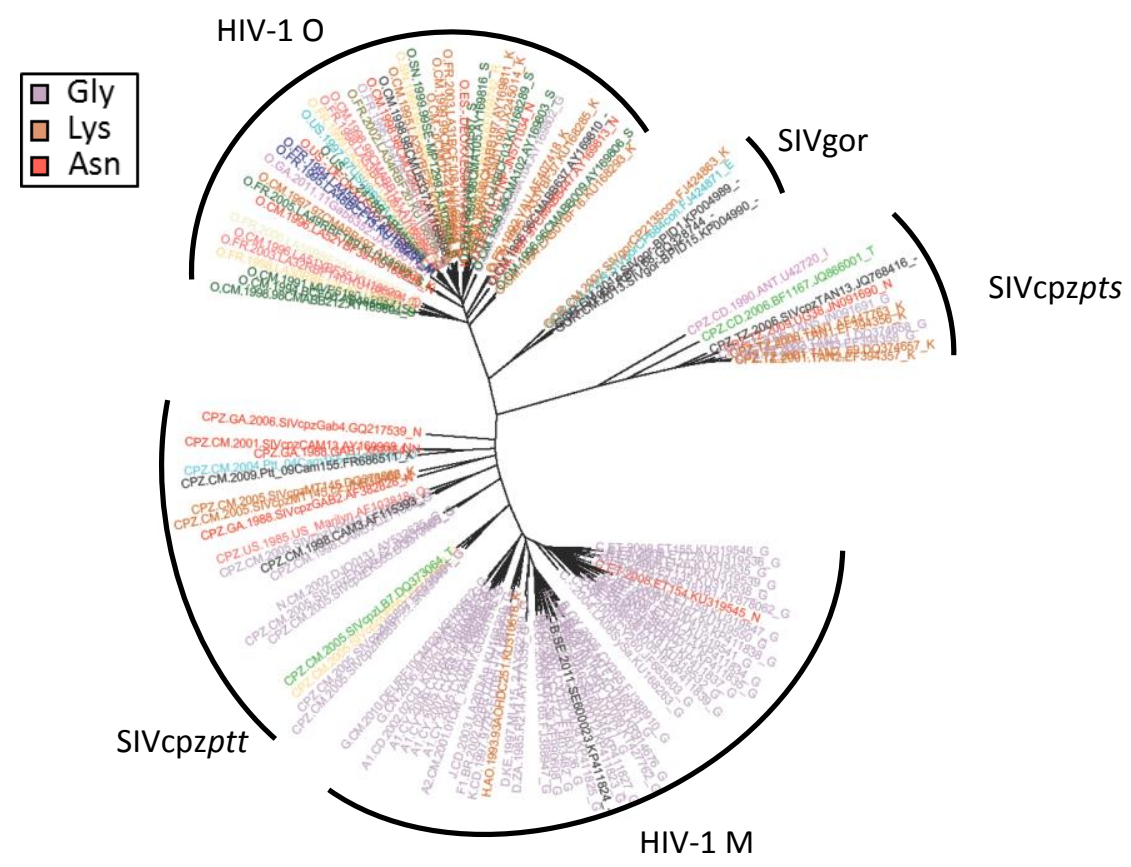

Figure 1. Projection of $\mathbf{E n v}_{458}$ on the HIV-1/SIV phylogeny. Each leaf (corresponding to an HIV/SIV strain) is color coded based on the amino acid present at Env 458 . The evolutionary rate of Env $_{458}$ was found to be slower in HIV-1 group $M$ than in the rest of the phylogeny.

Different rate deceleration patterns identified in different branches. A special focus was given

87 to rate deceleration events, as they likely indicate a gain of function in a protein. In Figure 2 we mapped the percent of rate shifts in each protein observed in each lineage, corresponding to different virus speciation events. While rate deceleration distribution patterns differed between

90 the different lineages (see Figure 2 and Supplementary Table 1), one common theme was that

91 all prominent branches underwent a significant portion of their rate deceleration events in the

92 Env protein. The next two common rate decelerating proteins were Gag and Pol identified in all

93 four highlighted branches, which is expected given that together with Env these are the longest

94 proteins in HIV/SIV. When controlling for protein size (as in Table 1), a different pattern

95 emerged: Vpu seems to have undergone a disproportionate portion of rate deceleration events,

96 in particular in the branch leading to group M. In general, the rate deceleration patterns for the

97 non-structural proteins seemed to be branch-specific: In the branch leading to SIVgor and group 
$98 \mathrm{O}, \mathrm{Nef}$ and Vif experienced the most relative rate deceleration events. In the branch leading to

99 group $M$, Vpu was responsible for the most rate deceleration events; and in the branch leading

100 to group O, Rev contributed a significant portion of all rate decelerations for that branch

101 (Supplementary Table 1). The branch separating SIVcpzpts from other viruses displayed a more

102 diverse pattern with relatively less rate decelerations in the Env protein and more rate

103 decelerations in the Nef, Vif and Vpu proteins.

104 Table 1. Rate shifts as percentage from total protein size for prominent branches, for rate

105 decelerating sites (upper) and rate accelerating sites (lower), colored by intensity. The raw

106 data underlying this table are provided in Supplementary File 2.

\begin{tabular}{|c|c|c|c|c|c|c|c|c|c|c|}
\hline DEC & \multicolumn{3}{|c|}{$\begin{array}{l}\text { Structural } \\
\text { proteins }\end{array}$} & \multicolumn{2}{|c|}{$\begin{array}{c}\text { Regulatory } \\
\text { proteins }\end{array}$} & \multicolumn{4}{|c|}{$\begin{array}{l}\text { Accessory } \\
\text { proteins }\end{array}$} & \multirow{2}{*}{$\begin{array}{c}\text { Total number of } \\
\text { mutations }\end{array}$} \\
\hline Branch/protein & Env & Gag & Pol & $\operatorname{Rev}$ & Tat & Nef & Vif & Vpr & Vpu & \\
\hline Group M & $1 \%$ & $1 \%$ & $1 \%$ & $1 \%$ & $0 \%$ & $0 \%$ & $0 \%$ & $0 \%$ & $5 \%$ & 28 \\
\hline Group 0 & $2 \%$ & $1 \%$ & $0 \%$ & $8 \%$ & $0 \%$ & $1 \%$ & $1 \%$ & $1 \%$ & $4 \%$ & 46 \\
\hline SIVgor+P+O & $0 \%$ & $0 \%$ & $0 \%$ & $0 \%$ & $0 \%$ & $2 \%$ & $1 \%$ & $0 \%$ & $0 \%$ & 14 \\
\hline SIVcpzpts & $1 \%$ & $1 \%$ & $0 \%$ & $0 \%$ & $0 \%$ & $3 \%$ & $2 \%$ & $1 \%$ & $5 \%$ & 27 \\
\hline ACC & & & & & & & & & & Total number of \\
\hline Branch/protein & Env & Gag & Pol & Rev & Tat & Nef & Vif & Vpr & Vpu & mutations \\
\hline Group M & $1 \%$ & $1 \%$ & $0 \%$ & $0 \%$ & $1 \%$ & $1 \%$ & $1 \%$ & $1 \%$ & $1 \%$ & 23 \\
\hline Group 0 & $3 \%$ & $2 \%$ & $1 \%$ & $3 \%$ & $0 \%$ & $1 \%$ & $2 \%$ & $0 \%$ & $1 \%$ & 51 \\
\hline SIVgor+P+O & $0 \%$ & $0 \%$ & $0 \%$ & $0 \%$ & $0 \%$ & $2 \%$ & $0 \%$ & $1 \%$ & $1 \%$ & 10 \\
\hline SIVcpzpts & $1 \%$ & $1 \%$ & $0 \%$ & $0 \%$ & $0 \%$ & $3 \%$ & $1 \%$ & $2 \%$ & $5 \%$ & 31 \\
\hline
\end{tabular}




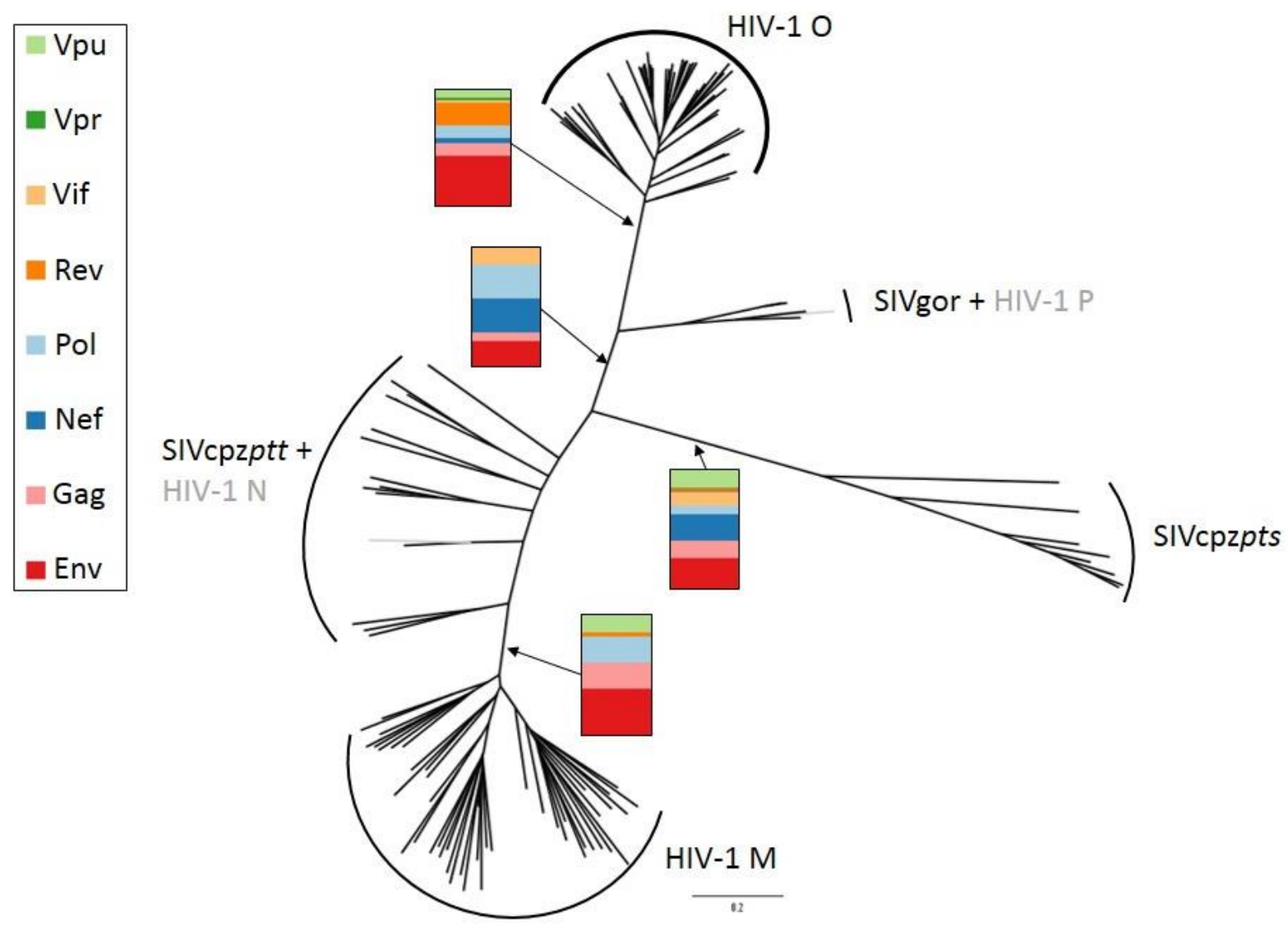

109 Figure 2. Proposed rate deceleration patterns in prominent branches, shown on the

110 phylogeny. Rate decelerations are shown as percentage from total rate decelerations for each 111 branch.

112 Parallel rate shift events in HIV-1 groups $\mathbf{M}$ and $\mathbf{0}$. Rate shift events common to several HIV-1

113 groups are of special interest, since they may better reflect the species barrier in general and

114 not lineage-specific adaptation event. As is evident from Table 2, most parallel rate shift events

115 of HIV-1 groups $\mathrm{O}$ and $\mathrm{M}$ (or their closest precursor branch) were rate deceleration events, half

116 of which occurred in the highly conserved Pol protein. Notably, the consensus amino acid

117 identity in those parallel-occurring rate deceleration events varied between the $\mathrm{M}$ and $\mathrm{O}$ clade

118 for all identified positions, suggesting once again that different adaptation strategies occurred in

119 each lineage. Only a single parallel rate acceleration event was identified, in $\mathrm{Nef}_{8}$ of HIV-1 
120 groups $\mathrm{M}$ and $\mathrm{O}$. This may indicate a relaxation of prior constraint as the SIV allele was

121 maintained in many HIV-1 sequences.

122 Table 2. Rate shifting sites identified independently in parallel in HIV-1 groups $M$ and $O$ or

123 their immediate ancestor.

\begin{tabular}{lllll}
\hline Protein & Rate Shifting site & Rate shift type & Branches & Consensus Amino Acids \\
\hline Pol & 349 & Deceleration & $M$, Pre O & M: E, Pre O: P \\
\hline Pol & 406 & Deceleration & Pre M, O & Pre M: S, O: V \\
\hline Pol & 926 & Deceleration & M, Pre O & M: K, Pre O: T \\
\hline Env & 61 & Deceleration & $M, O$ & M: Y, O: T \\
\hline Env & 772 & Deceleration & $M, O$ & M: R, O: S \\
\hline Rev & 71 & Deceleration & Pre M, O & Pre M: V, O: N \\
\hline Nef & 8 & Acceleration & $M, O$ & NA \\
\hline
\end{tabular}

$124 \quad$ NA- Not applicable

Rate shift events are often parallel to known macromolecular adaptation events. Intense

research into the origins of the HIV-1 pandemic and its sources identified several major adaptation events of HIV-1 to its human host. These events include adapting to cellular variations such as differences in host receptor CD4; overcoming host innate immune restriction factors such as Tetherin (BST-2) and APOBEC3G; and of course, evasion from the adaptive immune system [16, 20-25]. We therefore sought to evaluate whether the proposed rate shifts agree with the known adaptation events.

Anti Tetherin/BST-2 adaptations. Tetherin/BST-2 is a trans-membrane antiviral restriction factor that disrupts the budding phase of the retroviral lifecycle, thus preventing the infection from spreading $[26,27]$. SIV cpz and many more SIV's antagonize Tetherin through interactions of their nef protein with the cytoplasmic tail of Tetherin $[28,29]$. Tetherin is a fast evolving protein, and differs between chimpanzee, gorilla and human [30, 31]. In humans, a significant deletion in the cytoplasmic tail of Tetherin rendered it invulnerable to the SIV's Nefbased counteraction [29]. Therefore, in order to regain infectivity HIV-1 developed an 
139 alternative way to antagonize Tetherin. In groups M and N, Vpu protein adapted to antagonize

140 Tetherin through its transmembrane domain [32]. In group $\mathrm{O}$ the virus adapted its Nef protein

141 to counteract the human Tetherin, albeit with less efficiency than in group $M[33,34]$. Notably, a

142 single O strain that uses its Vpu protein to encounter human Tetherin has been described [35].

143 No anti-Tetherin adaptation event is known to have occurred in the rare group P [36]. In gorilla,

144 the Nef protein adapted to encounter its host's Tetherin but also maintained its ability to

145 encounter the chimpanzee tetherin [37, 38].

146 Our results support the described anti-Tetherin adaptation events: a dramatically high

147 proportion (14\% of all identified group $M$ rate decelerations) were found in the Vpu protein,

148 most of them in the Tetherin-binding domain (Supplementary Table 1 and Figure 3). In addition,

149 in HIV-1 group $M$ the Nef protein was identified with three rate accelerations, supporting the

150 loss of function of its anti-Tetherin activity. This is also supported by a reintroduction

151 experiment of HIV-1 group M strain into chimpanzee in which M-Nef readapted to chimpanzee

152 host, as the mutations required for M-Nef to regain anti-Tetherin activity in chimpanzees (Nef ${ }_{163}$

153 and $\mathrm{Nef}_{169}$ ) [39] were located proximally to $\mathrm{Nef}_{157}$, one of the rate accelerating sites of $\mathrm{M}-\mathrm{Nef}$

154 identified in our study. Numerous O-Nef rate acceleration events were found as well, supporting

155 the loss of chimpanzee/gorilla anti-Tetherin function. A preponderance of acceleration and

156 deceleration events were also found in the lineages leading to the ancestor of SIVgor and $\mathrm{O}$ and

157 to SIVcpzpts; one of those rate deceleration events is at $\mathrm{Nef}_{177}$, which is proximal to the C-loop

158 region which has been shown to be related to O-Nef anti-Tetherin activity [40]. Thus, our

159 analysis pinpoints the sites that are likely responsible for the dramatic changes in function of

160 Vpu and Nef throughout SIVcpz and HIV evolution. 
161

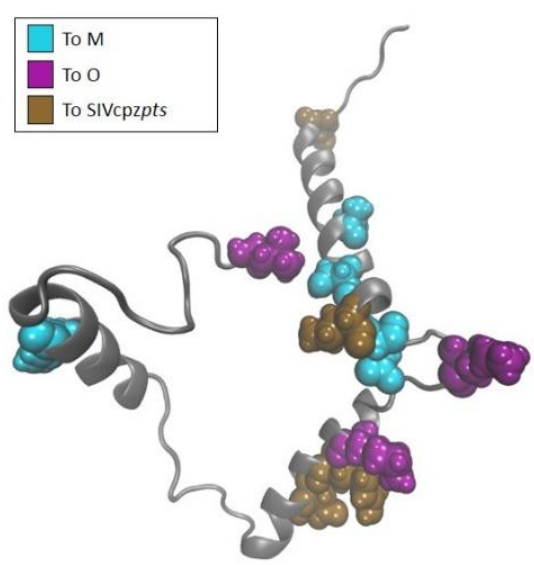

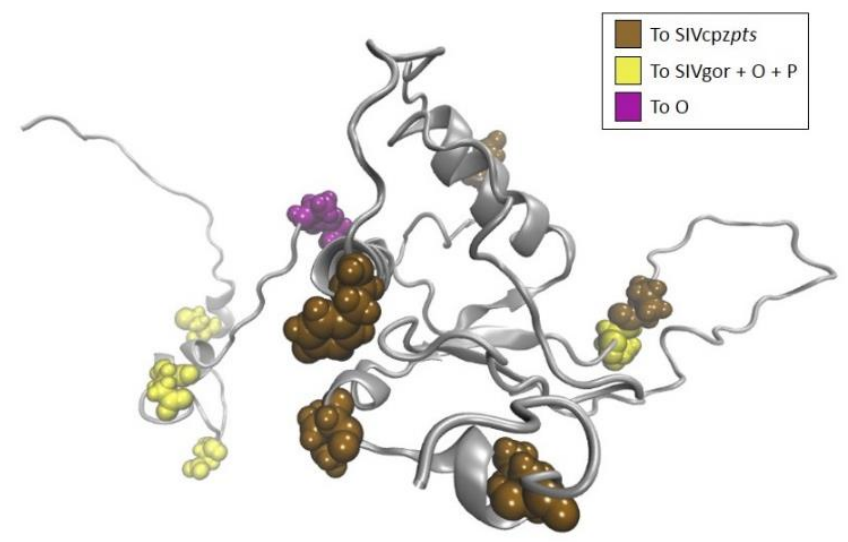

162 Figure 3. Projection of the identified rate decelerations onto the tertiary structure of Vpu 163 protein (left, PDB ID: 2N28) and Nef protein (right, obtained from [41]) in the major lineages 164 where it was identified.

Anti APOBEC3G adaptations. APOBEC3G (A3G) is a broad-range antiviral protein that is packaged into HIV-1 virions, and upon reverse transcription adds $\mathrm{C} \rightarrow \mathrm{U}$ mutations to the synthesized DNA strand, thus potentially creating nonviable genomes [42]. Lentiviruses use their Vif protein to overcome this factor by degrading it as well as other antiviral proteins from the APOBEC3 family (such as A3F, A3D) [43]. A3G variants of human, chimpanzee and gorilla have been studied and the Vif recognition domain has been identified at A3G residues 126-132 [44].

171 Of those species, gorilla has a different residue at A3G position 129 as compared to that of 172 human and chimpanzee A3Gs and indeed the SIVgor adaptation event to the different host A3G

173 has been demonstrated by $[45,46]$. It has been shown that SIVgor can replicate in the presence

174 of human-, gorilla- and chimpanzee-A3G, but HIV-1 group M or SIVcpz cannot replicate in the

175 presence of gorilla-A3G, indicative of a gain-of-function in the adaptation process of SIVgor to 176 gorilla [46]. In line with these findings, our analysis shows that the branch leading to SIVgor and HIV-

1781 groups $\mathrm{O}$ and $\mathrm{P}$ experienced $14 \%$ of its rate decelerations in the Vif protein, particularly in 
179 positions $\mathrm{Vif}_{73}$ and $\mathrm{Vif}_{167} . \mathrm{In} \mathrm{HIV}-1, \mathrm{Vif}_{73}$ is in close proximity to the A3G-binding motif $\mathrm{Vif}_{69-72}$ that

180 is also responsible in-part for interaction with APOBEC3F (which in itself contains differences in

181 the Vif interacting loop between chimpanzee and human/gorilla) [47-49]. O-Vif experienced a

182 single rate deceleration in position $\mathrm{Vif}_{127}$, which is located in the Cullin-5 interacting domain [48].

183 Lack of rate deceleration events in M-Vif suggest no adaptation of this protein in this strain to

184 the human host; this is supported by the observation that SIVcpz-Vif can encounter human A3G $185[46]$.

Env adaptations. Another major barrier for host jumps lies at the cell entry level. CD4 is

187 the target receptor of SIV and HIV, while CCR5 or CXCR4 act as co-receptors, recognized by the

188 viral Env protein which is exposed to the virus external surface. Previous studies mapped the

189 genetic diversity between different chimpanzees' CD4 and revealed that there are several

190 differences between chimpanzee subspecies as well as differences between chimpanzee versus

191 gorilla and human, especially in the regions that are in close contact with Env subunit gp120 [50,

192 51]. Some of those changes affect the glycosylation patterns of CD4, making these differences

193 even more distinguishable $[50,51]$. It is therefore likely that HIV-1 had to adapt to recognize

194 these differences in CD4.

Our analysis overall supports a high level of deceleration and acceleration events found

196 in Env, possibly reflecting the changes that occurred in the host CD4. For instance, many of the

197 changes in M-Env occurred in CD4 interacting residues of Env $374, E_{455}, E_{45} 46$ and Env 458 [52].

198 However, we cannot rule out that the very high rate of evolution in Env, driven by the adaptive

199 immune system [53], have led to changes that "mimic" a rate shift. Indeed when accounting for

200 the size of the Env protein, it seems that this protein is less pronounced in its rate shift

201 distribution (Table 1). 
$\mathbf{G a g}_{30}$ human adaptation marker. Position 30 of Gag polyprotein has been previously

203 suggested to be an adaptation event of HIV-1 to its human host, as it diversified from

204 Methionine in SIVcpzptt and SIVgor strains into Lysine or Arginine in many HIV-1 strains [54].

205 Reintroduction of HIV-1 to chimpanzees resulted in reversion of this position back to the

206 conserved Met of SIVcpzptt and therefore it was suggested as an adaptation event to the human

207 host [54]. Notably, subtype C of group M is conserved for Methionine at $\mathrm{Gag}_{30}$, suggesting that

208 adaptation occurred only in some subtypes.

209 Our results indeed suggest an evolutionary rate shift event in Gag $_{30}$ yet are not

210 conclusive about the branches in which the rate shift happened. This is likely since this site

211 displays a "content shift" rather than a strong "rate shift" (elaborated in the discussion).

212 Table 3. Summary of HIV rate deceleration events related to known antiviral activity.

\begin{tabular}{lclll}
\hline Viral Lineage & Viral Protein & $\begin{array}{l}\text { Rate } \\
\text { decelerating } \\
\text { positions } \\
\text { identified }\end{array}$ & $\begin{array}{l}\text { Rate deceleration(s) } \\
\text { related to the } \\
\text { following restriction } \\
\text { factor }\end{array}$ & Function of the restriction factor \\
\hline Group M & Vpu & $18,23,28,66$ & Tetherin & $\begin{array}{l}\text { Prevents virus release from infected } \\
\text { cells }\end{array}$ \\
\hline SIVgor and Group O Nef & $\begin{array}{l}20,28,38,49, \\
50,177\end{array}$ & Tetherin & $\begin{array}{l}\text { Prevents virus release from infected } \\
\text { cells }\end{array}$ \\
\hline SIVgor and group O Vif & $73,127,167$ & APOBEC3G & $\begin{array}{l}\text { Induces hypermutation of viral } \\
\text { genomes }\end{array}$ \\
\hline
\end{tabular}

213 Discussion

214 Understanding the molecular changes in a pathogen when adapting to infect a new host species

215 is of high importance. To the best of our knowledge, our study represents the first large-scale

216 approach to detect adaptation events in the transition from non-human primates to humans,

217 and relies on a robust phylogenetic modeling approach. We discovered many lineage-specific

218 adaptation-like events in many proteins of HIV-1, SIVgor and SIVcpzpts, and have reported sites

219 in where groups $\mathrm{M}$ and $\mathrm{O}$ suggested to undergone parallel rate shifts. The majority of sites 
220 undergoing rate deceleration were found in the Env protein; when correcting for protein size,

221 the relatively highest number of rate decelerating were found in proteins that adapted to host

222 restriction factors. Accordingly, we suggest that the major common barrier for a host species

223 jump is composed of both the entry stage and the stage where the virus must overcome the first

224 barrier of cellular defenses. Our results further support a differential model of adaptation:

225 groups $\mathrm{M}$ and $\mathrm{O}$ underwent adaptation in different proteins, and when adaptation occurred at

226 similar sites, the amino-acid was different in both groups. This model is supported by the fact

227 that activity of group $\mathrm{M}$ and $\mathrm{O}$ proteins are indeed different (e.g., Vpu), and by the fact that each

228 group originated from an SIV from a different primate.

229 Since group $M$ is the pandemic strain of HIV-1, we initially expected that it would experience the

230 highest number of rate shift events among all HIV-1 groups, indicating more efficient adaptation

231 to its human host. Surprisingly, our analysis revealed that group $\mathrm{O}$ had almost twice the amount

232 of rate shift events, despite being a non-pandemic strain that remained localized mainly to

233 infections in west-central Africa $[55,56]$. We suggest that the number of rate shift events is not

234 the determinant of a pandemic strain; it may rather reflect the relative height of the species

235 barrier a strain had to overcome, as chimpanzee from which group $M$ originated is genetically

236 closer to human than gorilla from which group O originated [57, 58].

237 We further noted a large number of rate shifts that occurred in the lineage separating the two

238 subspecies of SIVcpz (SIVcpzptt and SIVcpzpts). Presumably these events correspond to

239 adaptations of the virus to the two chimpanzee subspecies, which are genetically divergent.

240 Further research will be required to understand if and how this has affected the adaptation of

241 the virus to the human host. 
242 Limitations. This study has several limitations. First, the availability of full coverage of SIVcpz

243 and the SIVgor genomes is still low, reducing the statistical power of the analysis. Indeed, we

244 noted that increasing the sample size of group $M$ sequences that are much more available, led

245 to detection of more rate shifting sites (Supplementary File 1). We expect that the availability of

246 additional SIVgor sequences will increase the number of rate shift events that are unique in that

247 clade, possibly revealing more important sites for this lineage. Second, the method that we

248 utilized to identify rate shifts is calibrated to identify dramatic changes in the evolutionary rate

249 along a lineage. Accordingly, it cannot detect sites where a "content shift" occurred, i.e. the

250 amino acid changed and remained conserved in two complementary lineages, since this entails

251 only a minor change in evolutionary rate. This is partially demonstrated in $\mathrm{Gag}_{30}$, where the rate

252 of evolution changed mildly, while the content of this site changed between chimpanzee and

253 human viruses.

254 Implications. The compiled list of the positions suggested as rate decelerating can now serve as

255 a guide for future functional studies that aim to understand the differences among HIV-1 and

256 SIV proteins. Furthermore, the ability to track adaptation events by utilizing sequence data

257 highlights the power of the method when studying emerging pandemics, strengthening the

258 need to sequence full genomes of pathogens broadly.

259 Conclusions. Genetic sequences of viruses and specifically HIV-1 and SIV viruses can be

260 harnessed to identify adaptation events of emerging pathogens to their new host species. Our

261 results suggest that innate immunity serves as a strong barrier for cross-species transmission

262 events, and that this barrier imposed a strong selective pressure for viruses to adapt as they

263 crossed these barriers with increasing efficiency. 


\section{Methods}

265 In order to collect sequences for this study, the Los Alamos HIV sequence database (available

266 online at http://www.hiv.lanl.gov [19]) was queried for HIV-1 sequences from the same strain

267 that spanned all nine HIV-1 open reading frames (ORFs: gag, pol, vif, vpr, tat, rev, vpu, env and

$268 n e f)$ and for SIVcpz and SIVgor strains that spanned the corresponding ORFs (with the exception

269 of vpx). This led to 2004 sequences of HIV-1 group M, 45 sequences of HIV-1 group 0, 9

270 sequences of HIV-1 group N, 2 sequences of HIV-1 group P, 4 sequences of SIVgor and 29

271 sequences of SIVcpz. Two different datasets were constructed: (i) with a very large number of

272 group $\mathrm{M}$ sequences compared to the amount of group $\mathrm{O}$ sequences, and (ii) with an equal

273 number of group $\mathrm{M}$ and group $\mathrm{O}$ sequences. Due to computational reasons, dataset (i) included

274200 HIV-1 sequences, most of them group M. In both datasets, the sequences were sampled so

275 that the $\mathrm{n}$ most distant strains (in terms of genetic distance) were sampled. Due to extremely

276 high similarity, HIV-1 groups N and P sequences were reduced to a single representative strain

277 from each. The IIIB_LAI strain was added manually as a reference sequence. The results in this

278 study are reported mainly with datasets (ii), chosen since it allows comparing the result from

279 group $\mathrm{M}$ and group O. Additional sites found with datasets (i) are reported in Supplementary

$280 \quad$ File 1.

281 Initial multiple sequence alignments of the nine proteins were performed using PRANK and

282 iteratively improved until convergence [59]. In order to reconstruct of the phylogenetic

283 relationship between the sequences, we concatenated the alignments of Gag, Pol, Vif, Vpr, Tat

284 and Env and provided this as input for PhyML [60]. We next used the reconstructed phylogeny

285 as a guide tree to realign each protein with PRANK. JpHMM was used to validate that the strains

286 used in the analysis are not inter-group recombinants [61]. 
287 In order to identify evolutionary rate shifts, we used RASER [18] to analyze each of the nine

288 proteins separately, with the proteome-based phylogeny as input. RASER is a likelihood-based

289 phylogenetic method for detecting a change in site-specific evolutionary rates. First, a likelihood

290 ratio test against a null model of no rate shifts is performed in order to assess if a model

291 enabling rate shifts better fits the data. Next, the posterior probability of rate-shift is calculated

292 at each site and sites with a probability higher than 0.6 are considered here as significant.

293 Finally, for each such site, the method lists the lineages where the rate shift occurred with the

294 highest probability, and further categorizes each sites as undergoing either a rate-deceleration

295 or a rate-acceleration.

296 In order to test for sequence sampling effects on the identified rate shift patterns, we repeated

297 the analysis with increased amount of group $M$ sequences $(n=183)$, reduced amount of group 0

298 sequences $(n=13)$ and no SIVgor sequences, denoted as dataset (i). Analysis revealed more rate

299 decelerations in the branch leading to group $M$ than in dataset (ii) analysis (48 compared to 28,

300 Supplementary File 1). 54\% of the positions identified as rate-decelerating in group $\mathrm{M}$ in dataset

301 (ii) ( $n=15)$ were also identified in dataset (i). Chi-squared tests for differences in group M rate

302 shift distributions showed no significant difference $(p=0.11$ and 0.87 for rate decelerations and

303 accelerations, respectively), indicating that the patterns of rate shifts between the large group

304 M sample and the smaller group M sample are similar.

\section{Acknowledgements}

306 The authors would like to thank Dr. Osnat Penn, Prof. Eran Bacharach (Tel Aviv University) and

307 Prof. Tal Pupko (Tel Aviv University) for their contribution to the inception of this study, Prof.

308 Matthias Geyer (University of Bonn) for providing the Nef protein model structure, Dr. Tzachi

309 Hagai and Stern lab members for helpful comments and discussion. 
311 The authors declare that they have no competing interests involved with this study.

\section{Bibliography}

1. Hahn, B.H., G.M. Shaw, K.M. De Cock, and P.M. Sharp, AIDS - AIDS as a zoonosis: Scientific and public health implications. Science, 2000. 287(5453): p. 607-614.

2. Van Heuverswyn, F., Y. Li, C. Neel, E. Bailes, B.F. Keele, W. Liu, S. Loul, C. Butel, F. Liegeois, Y. Bienvenue, E.M. Ngolle, P.M. Sharp, G.M. Shaw, E. Delaporte, B.H. Hahn, and M. Peeters, Human immunodeficiency viruses: SIV infection in wild gorillas. Nature, 2006. 444(7116): p. 164.

3. D'Arc, M., et al., Origin of the HIV-1 group O epidemic in western lowland gorillas. Proc Natl Acad Sci U S A, 2015. 112(11): p. E1343-52.

4. Takehisa, J., M.H. Kraus, A. Ayouba, E. Bailes, F. Van Heuverswyn, J.M. Decker, Y. Li, R.S. Rudicell, G.H. Learn, C. Neel, E.M. Ngole, G.M. Shaw, M. Peeters, P.M. Sharp, and B.H. Hahn, Origin and biology of simian immunodeficiency virus in wild-living western gorillas. J Virol, 2009. 83(4): p. 1635-48.

5. Bell, S.M. and T. Bedford, Modern-day SIV viral diversity generated by extensive recombination and cross-species transmission. PLoS Pathog, 2017. 13(7): p. e1006466.

6. Korber, B., M. Muldoon, J. Theiler, F. Gao, R. Gupta, A. Lapedes, B.H. Hahn, S. Wolinsky, and T. Bhattacharya, Timing the ancestor of the HIV-1 pandemic strains. Science, 2000. 288(5472): p. 1789-96.

7. Lemey, P., O.G. Pybus, A. Rambaut, A.J. Drummond, D.L. Robertson, P. Roques, M. Worobey, and A.M. Vandamme, The molecular population genetics of HIV-1 group 0. Genetics, 2004. 167(3): p. 1059-68.

8. Wertheim, J.O. and M. Worobey, Dating the Age of the SIV Lineages That Gave Rise to HIV-1 and HIV-2. Plos Computational Biology, 2009. 5(5): p. e1000377.

9. Bailes, E., F. Gao, F. Bibollet-Ruche, V. Courgnaud, M. Peeters, P.A. Marx, B.H. Hahn, and P.M. Sharp, Hybrid origin of SIV in chimpanzees. Science, 2003. 300(5626): p. 1713.

10. Leitner, T., M.-C. Dazza, M. Ekwalanga, C. Apetrei, and S. Saragosti, Sequence Diversity among Chimpanzee Simian Immunodeficiency Viruses (SIVcpz) Suggests that SIVcpz Pts Was Derived from SIVcpz Ptt through Additional Recombination Events. AIDS research and human retroviruses, 2007. 23(9): p. 1114-1118.

11. Li, Y., et al., Eastern chimpanzees, but not bonobos, represent a simian immunodeficiency virus reservoir. J Virol, 2012. 86(19): p. 10776-91.

12. Etienne, L., B.H. Hahn, P.M. Sharp, F.A. Matsen, and M. Emerman, Gene loss and adaptation to hominids underlie the ancient origin of HIV-1. Cell Host Microbe, 2013. 14(1): p. 85-92.

13. Longdon, B., M.A. Brockhurst, C.A. Russell, J.J. Welch, and F.M. Jiggins, The evolution and genetics of virus host shifts. PLoS Pathog, 2014. 10(11): p. e1004395.

14. Kuiken, T., E.C. Holmes, J. McCauley, G.F. Rimmelzwaan, C.S. Williams, and B.T. Grenfell, Host species barriers to influenza virus infections. Science, 2006. 312(5772): p. 394-7.

351 15. Taubenberger, J.K. and J.C. Kash, Influenza virus evolution, host adaptation, and pandemic formation. Cell Host Microbe, 2010. 7(6): p. 440-51. 
16. Parrish, C.R., E.C. Holmes, D.M. Morens, E.-C. Park, D.S. Burke, C.H. Calisher, C.A. Laughlin, L.J. Saif, and P. Daszak, Cross-species virus transmission and the emergence of new epidemic diseases. Microbiology and Molecular Biology Reviews, 2008. 72(3): p. 457-470.

17. Knudsen, B. and M.M. Miyamoto, A likelihood ratio test for evolutionary rate shifts and functional divergence among proteins. Proc Natl Acad Sci U S A, 2001. 98(25): p. 145127.

18. Penn, O., A. Stern, N.D. Rubinstein, J. Dutheil, E. Bacharach, N. Galtier, and T. Pupko, Evolutionary modeling of rate shifts reveals specificity determinants in HIV-1 subtypes. PLoS Comput Biol, 2008. 4(11): p. e1000214.

19. Kuiken, C., B. Foley, T. Leitner, C. Apetrei, B. Hahn, I. Mizrachi, J. Mullins, A. Rambaut, S. Wolinsky, and B. Korber, HIV sequence compendium 2010. 2010, Los Alamos National Laboratory (LANL), Los Alamos, NM (United States).

20. Sironi, M., R. Cagliani, D. Forni, and M. Clerici, Evolutionary insights into host-pathogen interactions from mammalian sequence data. Nature Reviews Genetics, 2015. 16: p. 224.

21. McLaren, P.J. and M. Carrington, The impact of host genetic variation on infection with HIV-1. Nature Immunology, 2015. 16: p. 577.

22. Kirchhoff, F., Immune evasion and counteraction of restriction factors by HIV-1 and other primate lentiviruses. Cell Host Microbe, 2010. 8(1): p. 55-67.

23. Kluge, S.F., D. Sauter, and F. Kirchhoff, SnapShot: antiviral restriction factors. Cell, 2015. 163(3): p. 774-774. e1.

24. Kaslow, R.A., M. Carrington, R. Apple, L. Park, A. Munoz, A. Saah, J.J. Goedert, C. Winkler, S.J. O'Brien, and C. Rinaldo, Influence of combinations of human major histocompatibility complex genes on the course of HIV-1 infection. Nature medicine, 1996. 2(4): p. 405-411.

25. Simon, V., N. Bloch, and N.R. Landau, Intrinsic host restrictions to HIV-1 and mechanisms of viral escape. Nature Immunology, 2015. 16: p. 546.

26. Neil, S.J.D., T. Zang, and P.D. Bieniasz, Tetherin inhibits retrovirus release and is antagonized by HIV-1 Vpu. Nature, 2008. 451(7177): p. 425-U1.

27. Van Damme, N., D. Goff, C. Katsura, R.L. Jorgenson, R. Mitchell, M.C. Johnson, E.B. Stephens, and J. Guatelli, The interferon-induced protein BST-2 restricts HIV-1 release and is downregulated from the cell surface by the viral Vpu protein. Cell host \& microbe, 2008. 3(4): p. 245-252.

28. Jia, B., R. Serra-Moreno, W. Neidermyer, A. Rahmberg, J. Mackey, I.B. Fofana, W.E. Johnson, S. Westmoreland, and D.T. Evans, Species-specific activity of SIV Nef and HIV-1 Vpu in overcoming restriction by tetherin/BST2. PLoS Pathog, 2009. 5(5): p. e1000429.

29. Zhang, F., S.J. Wilson, W.C. Landford, B. Virgen, D. Gregory, M.C. Johnson, J. Munch, F. Kirchhoff, P.D. Bieniasz, and T. Hatziioannou, Nef proteins from simian immunodeficiency viruses are tetherin antagonists. Cell Host Microbe, 2009. 6(1): p. 5467.

30. McNatt, M.W., T. Zang, T. Hatziioannou, M. Bartlett, I.B. Fofana, W.E. Johnson, S.J. Neil, and P.D. Bieniasz, Species-specific activity of HIV-1 Vpu and positive selection of tetherin transmembrane domain variants. PLoS Pathog, 2009. 5(2): p. e1000300.

31. Evans, D.T., R. Serra-Moreno, R.K. Singh, and J.C. Guatelli, BST-2/tetherin: a new component of the innate immune response to enveloped viruses. Trends Microbiol, 2010. 18(9): p. 388-96. 
400

401

402

403

404

405

406

407

408

409

410

411

412

413

414

415

416

417

418

419

420

421

422

423

424

425

426

427

428

429

430

431

432

433

434

435

436

437

438

439

440

441

442

443

444

445

446

447

32. Sauter, D., et al., Tetherin-driven adaptation of Vpu and Nef function and the evolution of pandemic and nonpandemic HIV-1 strains. Cell Host Microbe, 2009. 6(5): p. 409-21.

33. Kluge, S.F., K. Mack, S.S. Iyer, F.M. Pujol, A. Heigele, G.H. Learn, S.M. Usmani, D. Sauter, S. Joas, D. Hotter, F. Bibollet-Ruche, L.J. Plenderleith, M. Peeters, M. Geyer, P.M. Sharp, O.T. Fackler, B.H. Hahn, and F. Kirchhoff, Nef proteins of epidemic HIV-1 group O strains antagonize human tetherin. Cell Host Microbe, 2014. 16(5): p. 639-50.

34. Bego, M.G., L. Cong, K. Mack, F. Kirchhoff, and E.A. Cohen, Differential Control of BST2 Restriction and Plasmacytoid Dendritic Cell Antiviral Response by Antagonists Encoded by HIV-1 Group M and O Strains. J Virol, 2016. 90(22): p. 10236-10246.

35. Mack, K., K. Starz, D. Sauter, S. Langer, F. Bibollet-Ruche, G.H. Learn, C.M. Sturzel, M. Leoz, J.C. Plantier, M. Geyer, B.H. Hahn, and F. Kirchhoff, Efficient Vpu-Mediated Tetherin Antagonism by an HIV-1 Group O Strain. J Virol, 2017. 91(6): p. e02177-16.

36. Sauter, D., S. Hué, S.J. Petit, J.-C. Plantier, G.J. Towers, F. Kirchhoff, and R.K. Gupta, HIV-1 Group $P$ is unable to antagonize human tetherin by Vpu, Env or Nef. Retrovirology, 2011. 8(1): p. 103.

37. Lim, E.S., H.S. Malik, and M. Emerman, Ancient adaptive evolution of tetherin shaped the functions of Vpu and Nef in human immunodeficiency virus and primate lentiviruses. J Virol, 2010. 84(14): p. 7124-34.

38. Yang, S.J., L.A. Lopez, C.M. Exline, K.G. Haworth, and P.M. Cannon, Lack of adaptation to human tetherin in HIV-1 group $O$ and P. Retrovirology, 2011. 8(1): p. 78.

39. Gotz, N., D. Sauter, S.M. Usmani, J.V. Fritz, C. Goffinet, A. Heigele, M. Geyer, F. BibolletRuche, G.H. Learn, O.T. Fackler, B.H. Hahn, and F. Kirchhoff, Reacquisition of NefMediated Tetherin Antagonism in a Single In Vivo Passage of HIV-1 through Its Original Chimpanzee Host. Cell Host \& Microbe, 2012. 12(3): p. 373-380.

40. Mack, K., Counteraction of the antiviral factor tetherin by HIV-1 group 0. 2017, Universität Ulm.

41. Geyer, M. and B.M. Peterlin, Domain assembly, surface accessibility and sequence conservation in full length HIV-1 Nef. FEBS Lett, 2001. 496(2-3): p. 91-5.

42. Mangeat, B., P. Turelli, G. Caron, M. Friedli, L. Perrin, and D. Trono, Broad antiretroviral defence by human $A P O B E C 3 G$ through lethal editing of nascent reverse transcripts. Nature, 2003. 424(6944): p. 99-103.

43. Kato, Y., R. Habas, Y. Katsuyama, A.M. Naar, and X. He, A component of the ARC/Mediator complex required for TGF beta/Nodal signalling. Nature, 2002. 418(6898): p. 641-6.

44. Russell, R.A., J. Smith, R. Barr, D. Bhattacharyya, and V.K. Pathak, Distinct Domains within APOBEC3G and APOBEC3F Interact with Separate Regions of Human Immunodeficiency Virus Type 1 Vif. Journal of Virology, 2009. 83(4): p. 1992-2003.

45. Compton, A.A. and M. Emerman, Convergence and divergence in the evolution of the APOBEC3G-Vif interaction reveal ancient origins of simian immunodeficiency viruses. PLoS Pathog, 2013. 9(1): p. e1003135.

46. Letko, M., G. Silvestri, B.H. Hahn, F. Bibollet-Ruche, O. Gokcumen, V. Simon, and M. Ooms, Vif Proteins from Diverse Primate Lentiviral Lineages Use the Same Binding Site in APOBEC3G. Journal of Virology, 2013. 87(21): p. 11861-11871.

47. Pery, E., K.S. Rajendran, A.J. Brazier, and D. Gabuzda, Regulation of APOBEC3 proteins by a novel YXXL motif in human immunodeficiency virus type 1 Vif and simian immunodeficiency virus SIVagm Vif. J Virol, 2009. 83(5): p. 2374-81.

48. Feng, Y., T.T. Baig, R.P. Love, and L. Chelico, Suppression of APOBEC3-mediated restriction of HIV-1 by Vif. Front Microbiol, 2014. 5: p. 450. 
49. Letko, M., T. Booiman, N. Kootstra, V. Simon, and M. Ooms, Identification of the HIV-1 Vif and Human APOBEC3G Protein Interface. Cell Rep, 2015. 13(9): p. 1789-99.

50. Hvilsom, C., F. Carlsen, H.R. Siegismund, S. Corbet, E. Nerrienet, and A. Fomsgaard, Genetic subspecies diversity of the chimpanzee CD4 virus-receptor gene. Genomics, 2008. 92(5): p. 322-8.

51. Brown, K.E., Parallel adaptation of CD4 in SIV-endemic Gorilla and Pan lineages. 2014, University at Albani.

52. Zhou, T., I. Georgiev, X. Wu, Z.Y. Yang, K. Dai, A. Finzi, Y.D. Kwon, J.F. Scheid, W. Shi, L. Xu, Y. Yang, J. Zhu, M.C. Nussenzweig, J. Sodroski, L. Shapiro, G.J. Nabel, J.R. Mascola, and P.D. Kwong, Structural basis for broad and potent neutralization of HIV-1 by antibody VRC01. Science, 2010. 329(5993): p. 811-7.

53. Yamaguchi-Kabata, Y. and T. Gojobori, Reevaluation of amino acid variability of the human immunodeficiency virus type 1 gp120 envelope glycoprotein and prediction of new discontinuous epitopes. J Virol, 2000. 74(9): p. 4335-50.

54. Wain, L.V., E. Bailes, F. Bibollet-Ruche, J.M. Decker, B.F. Keele, F. Van Heuverswyn, Y. Li, J. Takehisa, E.M. Ngole, G.M. Shaw, M. Peeters, B.H. Hahn, and P.M. Sharp, Adaptation of HIV-1 to its human host. Mol Biol Evol, 2007. 24(8): p. 1853-60.

55. Peeters, M., A. Gueye, S. Mboup, F. Bibollet-Ruche, E. Ekaza, C. Mulanga, R. Ouedrago, R. Gandji, P. Mpele, G. Dibanga, B. Koumare, M. Saidou, E. Esu-Williams, J.P. Lombart, W. Badombena, N. Luo, M. Vanden Haesevelde, and E. Delaporte, Geographical distribution of HIV-1 group O viruses in Africa. AIDS, 1997. 11(4): p. 493-8.

56. Leoz, M., F. Feyertag, A. Kfutwah, P. Mauclere, G. Lachenal, F. Damond, F. De Oliveira, V. Lemee, F. Simon, D.L. Robertson, and J.C. Plantier, The Two-Phase Emergence of Non Pandemic HIV-1 Group O in Cameroon. PLoS Pathog, 2015. 11(8): p. e1005029.

57. Hasegawa, M., H. Kishino, and T. Yano, Dating of the human-ape splitting by a molecular clock of mitochondrial DNA. J Mol Evol, 1985. 22(2): p. 160-74.

58. Ruvolo, M., Molecular phylogeny of the hominoids: inferences from multiple independent DNA sequence data sets. Mol Biol Evol, 1997. 14(3): p. 248-65.

59. Loytynoja, A. and N. Goldman, An algorithm for progressive multiple alignment of sequences with insertions. Proceedings of the National Academy of Sciences of the United States of America, 2005. 102(30): p. 10557-10562.

60. Guindon, S., J.F. Dufayard, V. Lefort, M. Anisimova, W. Hordijk, and O. Gascuel, New algorithms and methods to estimate maximum-likelihood phylogenies: assessing the performance of PhyML 3.0. Syst Biol, 2010. 59(3): p. 307-21.

61. Schultz, A.K., M. Zhang, I. Bulla, T. Leitner, B. Korber, B. Morgenstern, and M. Stanke, jpHMM: improving the reliability of recombination prediction in HIV-1. Nucleic Acids Res, 2009. 37(Web Server issue): p. W647-51. 

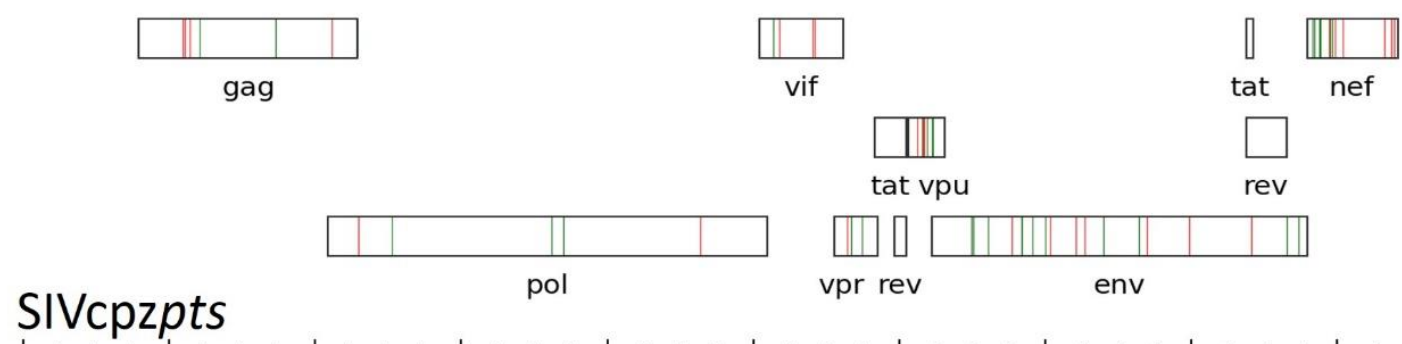

env
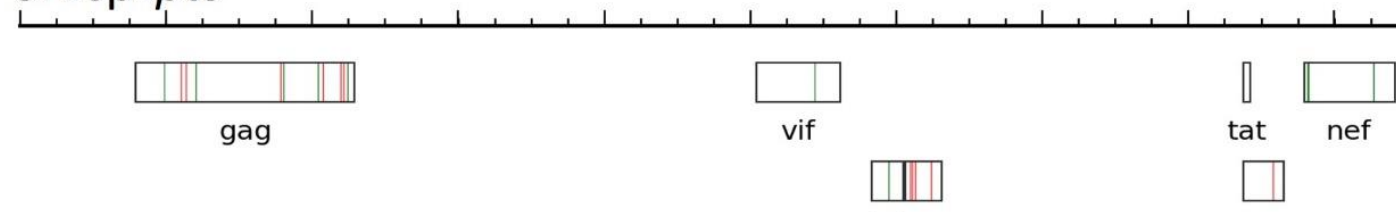

tat vpu

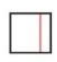

rev

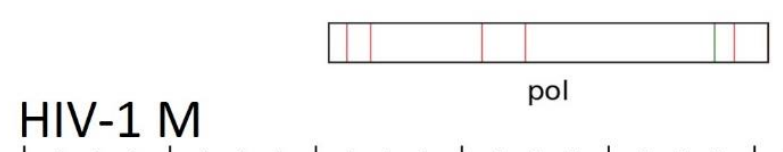

vpr rev

env

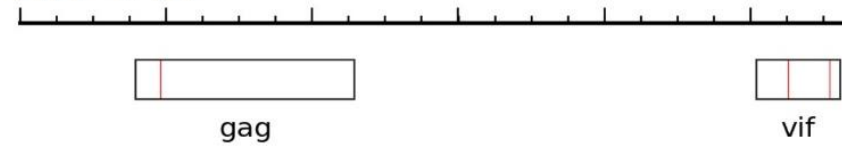

gag

SIVgor $+\mathrm{O}+\mathrm{P}$

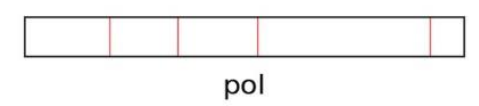

tat vpu

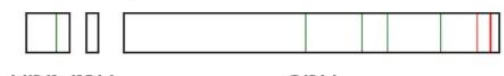

vpr rev

env

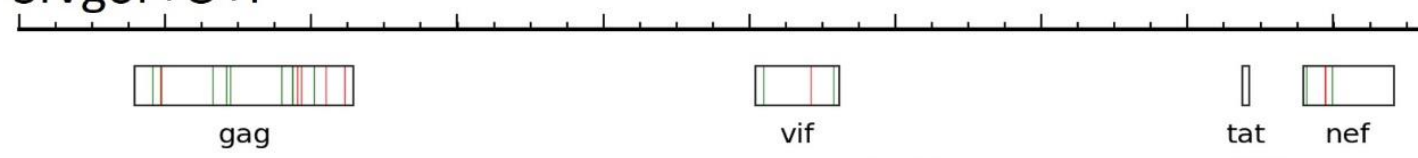

gag
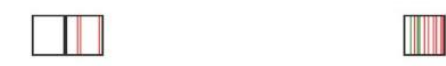

tat vpu

rev

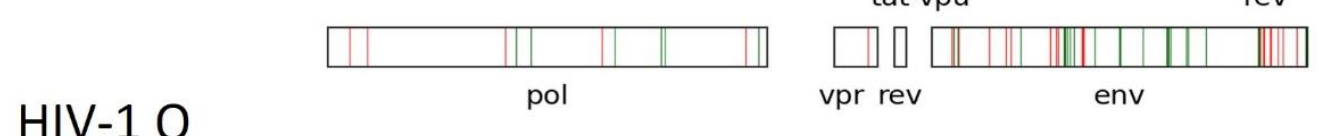

HIV-1 O

vpr rev

env

\begin{tabular}{llllllllllll}
\hline & 1000 & 2000 & 3000 & 4000 & 5000 & 6000 & 7000 & 8000 & 9000
\end{tabular}

491 Supplementary Figure 1. Proposed rate deceleration patterns in prominent branches shown

492 along the genome. Data shown in HXB2 coordinates. 
bioRxiv preprint doi: https://doi.org/10.1101/190769; this version posted January 18,2018 . The copyright holder for this preprint (which was not certified by peer review) is the author/funder, who has granted bioRxiv a license to display the preprint in perpetuity. It is made available under aCC-BY-NC-ND 4.0 International license.

494

495

496

497

498

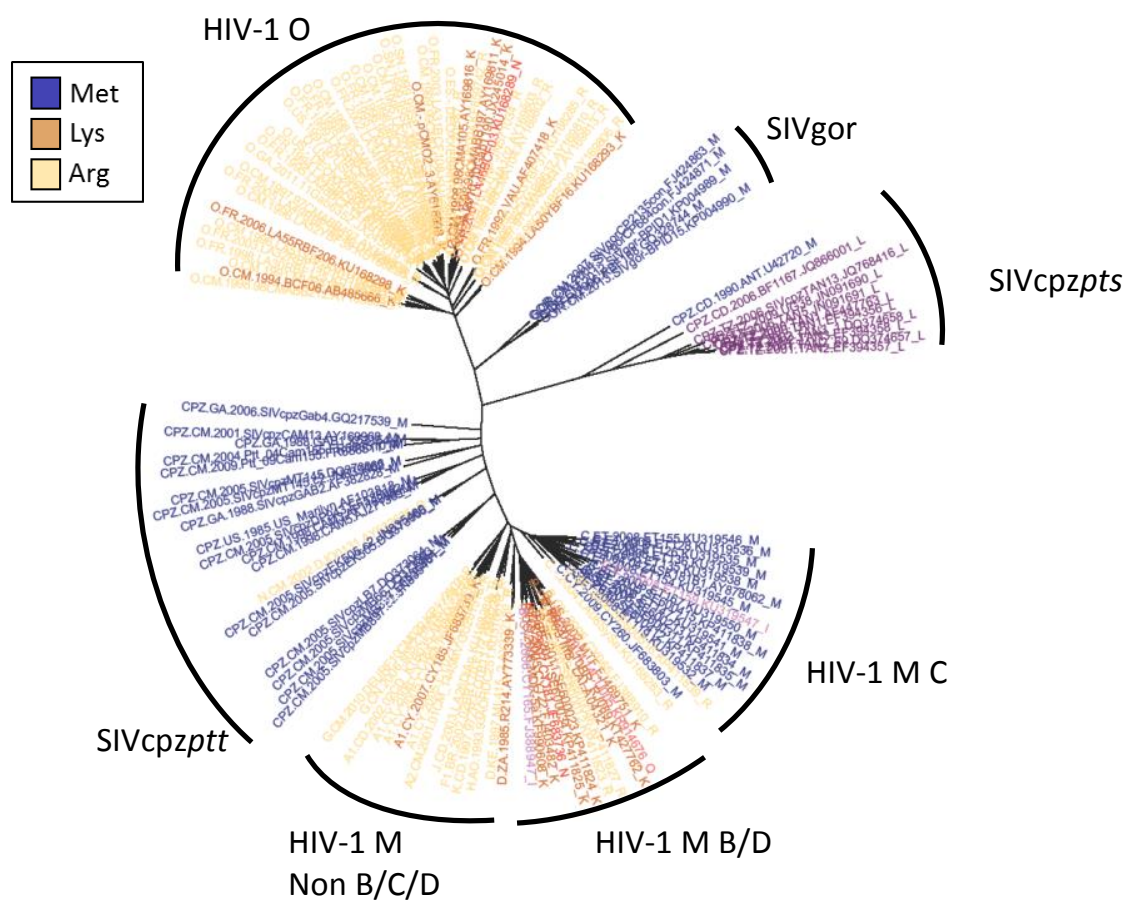

Supplementary Figure 2. Residues distribution for $\mathrm{Gag}_{30}$ along the HIV-1/SIV phylogeny. This position exhibits a pattern more similar to "content shift" than to "rate shift". 
500 branches, for rate decelerating sites (upper) and rate accelerating sites (lower), colored by

501 intensity.

DEC

\begin{tabular}{|c|c|c|c|c|c|c|c|c|c|}
\hline \multicolumn{10}{|l|}{ DEC } \\
\hline Branch/protein & Env & Gag & Pol & $\operatorname{Rev}$ & Tat & Nef & Vif & Vpr & Vpu \\
\hline Group M & $39 \%$ & $21 \%$ & $21 \%$ & $4 \%$ & $0 \%$ & $0 \%$ & $0 \%$ & $0 \%$ & $14 \%$ \\
\hline Group 0 & $43 \%$ & $11 \%$ & $11 \%$ & $20 \%$ & $0 \%$ & $4 \%$ & $2 \%$ & $2 \%$ & $7 \%$ \\
\hline SIVgor+P+O & $21 \%$ & $7 \%$ & $29 \%$ & $0 \%$ & $0 \%$ & $29 \%$ & $14 \%$ & $0 \%$ & $0 \%$ \\
\hline SIVcpzpts & $26 \%$ & $15 \%$ & $7 \%$ & $0 \%$ & $0 \%$ & $22 \%$ & $11 \%$ & $4 \%$ & $15 \%$ \\
\hline \multicolumn{10}{|l|}{ ACC } \\
\hline Branch/protein & Env & Gag & Pol & $\operatorname{Rev}$ & Tat & Nef & Vif & Vpr & Vpu \\
\hline Group M & $43 \%$ & $22 \%$ & $4 \%$ & $0 \%$ & $4 \%$ & $13 \%$ & $4 \%$ & $4 \%$ & $4 \%$ \\
\hline Group 0 & $55 \%$ & $18 \%$ & $12 \%$ & $6 \%$ & $0 \%$ & $4 \%$ & $4 \%$ & $0 \%$ & $2 \%$ \\
\hline SIVgor+P+O & $40 \%$ & $0 \%$ & $0 \%$ & $0 \%$ & $0 \%$ & $40 \%$ & $0 \%$ & $10 \%$ & $10 \%$ \\
\hline SIVcpzpts & $35 \%$ & $10 \%$ & $10 \%$ & $0 \%$ & $0 \%$ & $23 \%$ & $3 \%$ & $6 \%$ & $13 \%$ \\
\hline
\end{tabular}

ACC

Branch/protein

\begin{tabular}{|c|c|c|c|c|c|c|c|c|}
\hline Env & Gag & Pol & $\operatorname{Rev}$ & Tat & Nef & Vif & Vpr & Vpu \\
\hline $39 \%$ & $21 \%$ & $21 \%$ & $4 \%$ & $0 \%$ & $0 \%$ & $0 \%$ & $0 \%$ & $14 \%$ \\
\hline $43 \%$ & $11 \%$ & $11 \%$ & $20 \%$ & $0 \%$ & $4 \%$ & $2 \%$ & $2 \%$ & $7 \%$ \\
\hline $21 \%$ & $7 \%$ & $29 \%$ & $0 \%$ & $0 \%$ & $29 \%$ & $14 \%$ & $0 \%$ & $0 \%$ \\
\hline $26 \%$ & $15 \%$ & $7 \%$ & $0 \%$ & $0 \%$ & $22 \%$ & $11 \%$ & $4 \%$ & $15 \%$ \\
\hline Env & Gag & Pol & $\operatorname{Rev}$ & Tat & Nef & Vif & Vpr & Vpu \\
\hline $43 \%$ & $22 \%$ & $4 \%$ & $0 \%$ & $4 \%$ & $13 \%$ & $4 \%$ & $4 \%$ & $4 \%$ \\
\hline $55 \%$ & $18 \%$ & $12 \%$ & $6 \%$ & $0 \%$ & $4 \%$ & $4 \%$ & $0 \%$ & $2 \%$ \\
\hline $40 \%$ & $0 \%$ & $0 \%$ & $0 \%$ & $0 \%$ & $40 \%$ & $0 \%$ & $10 \%$ & $10 \%$ \\
\hline $35 \%$ & $10 \%$ & $10 \%$ & $0 \%$ & $0 \%$ & $23 \%$ & $3 \%$ & $6 \%$ & $13 \%$ \\
\hline
\end{tabular}

Group M

Group 0

SIVgor+P+O

SIVcpzpts

Supplementary Table 2. Maximum log-likelihood (LL) values for the analysis of the nine HIV$1 /$ SIVcpz/SIVgor proteins under the rate shift and null models.

\begin{tabular}{lllll}
\hline Protein & Rate Shift Model LL & Null Model LL & $2 \Delta L L$ & P-value $\left(\chi^{2}{ }^{2}\right)$ \\
\hline Gag & $-29,478.3$ & $-29,671.4$ & 386 & $<10^{-50}$ \\
\hline Pol & $-40,813.1$ & $-41,097.2$ & 568 & $<10^{-100}$ \\
\hline Vif & $-12,677.6$ & $-12,756.3$ & 157 & $<10^{-30}$ \\
\hline Vpr & $-5,328.1$ & $-5,355.76$ & 55 & $<10^{-10}$ \\
\hline Tat & $-8,918.23$ & $-8,947.61$ & 59 & $<10^{-10}$ \\
\hline Rev & $-12,662$ & & & \\
\hline Vpu & $-10,548.7$ & $-12,733.6$ & 143 & $<10^{-30}$ \\
\hline Env & $-92,347.6$ & $-10,664.1$ & 231 & $<10^{-50}$ \\
\hline Nef & $-15,611.4$ & $-15,804.1$ & 385 & $<10^{-50}$ \\
\hline
\end{tabular}


509 Supplementary File 1.

510 HIV/SIV sites identified as rate shifting for dataset (i). Positions are provided in HXB2 reference

511 sequence coordinates. Branch number field refer to the branch number outputted by RASER

512 [18]; for ease of reading we provide branch labels for most branches.

\section{Supplementary File 2.}

514 HIV/SIV sites identified as rate shifting for dataset (ii). Positions are provided in HXB2 reference

515 sequence coordinates. Branch number field refer to the branch number outputted by RASER

516 [18]; for ease of reading we provide branch labels for most branches. 